\title{
For older adults with hematologic malignancies, a comprehensive geriatric
}

\section{assessment matters}

\section{Raul Cordoba}

\author{
Fundacion Jimenez Diaz University Hospital, Health Research Institute IIS-FJD, Madrid, Spain \\ E-mail: RAUL CORDOBA - raul.cordoba@fjd.es
}

doi:10.3324/haematol.2021.279927

$I^{n}$ $\mathrm{n}$ this issue of Haematologica, DuMontier et al. address a key question in the management of older adults with hematologic malignancies by reporting the results of a randomized controlled trial of geriatric consultation plus standard care versus standard care alone. ${ }^{1}$ Cancer can be considered an age-related disease because the incidence of most cancers increases with age. ${ }^{2}$ With regards to hematologic malignancies, incidence rates increase for non-Hodgkin lymphoma, multiple myeloma, and acute myeloid leukemia, and remain relatively stable for acute lymphoblastic leukemia, chronic lymphocytic leukemia, and chronic myeloid leukemia among adults aged $\geq 75$ years. In spite of improving supportive care, survival for patients aged $\geq 75$ years with hematologic malignancies is generally poor, particularly for those with acute leukemia. Understanding the heterogeneity in the outcomes of patients with hematologic malignancies, as well as the treatment challenges and management of frailty and comorbidities among older patients may help physicians to better address the hematologic cancer burden and mortality in the aging population. ${ }^{3}$

Hematologic malignancies are a miscellaneous group of diseases with regard to biology, prognosis and treatment options. Treatment decisions in older patients should not only be influenced by disease characteristics such as stage, histology, cytogenetics, molecular markers, etc. but also by patient-related factors such as fitness, frailty, and patients' preferences. Furthermore, fitness and frailty are not static, but dynamic factors that may improve or deteriorate over time in the course of a disease and its treatment. Geriatric assessment is considered an important task during the diagnostic work-up and prior to deciding treatment in older adults with hematologic malignancies. ${ }^{4,5}$ Geriatric assessment includes a careful assessment of various domains including instrumental and basal activities of daily living (IADL, ADL), mobility, nutrition, cognitive function, and mental status. Many instruments, including screening tools (e.g., G8) and hematology-specific approaches (e.g., the brief Geriatric Assessment in Hematology tool, the GAH scale) have been suggested to perform geriatric assessments in patients with hematologic malignancies. ${ }^{6}$ A commonly accepted concept is to categorize older patients with hematologic malignancies into 'fit' for standard treatment, 'unfit' for attenuated treatment, and 'frail'/terminally ill, not suitable for specific hematologic therapies but best supportive care.

The study by DuMontier et al. presents the first randomzied controlled trial of geriatric consultation in older adults with hematologic malignancies. While the study did not meet its primary endpoint of improvement in survival, consultation did increase the proportion of patients who participated in a goals-of-care discussion. The study is important to the field of hematology as it is the first randomized controlled trial of its kind in hematology, in contrast to four separate randomized controlled trials enrolling older patients with solid tumors presented at the American Society of Clinical Oncology (ASCO) anual meeting in 2020. The primary outcome was 1-year overall survival and secondary endpoints included unplanned care utilization within 6 months of follow-up and documented end-of-life goals-of-care discussions. Patients who were assigned to the intervention group received simultaneously geriatric consultation with a geriatrician in addition to their standard oncologic care. Patients were assessed following the ASCO's Guideline for Geriatric Oncology for function and falls, comorbidity and polypharmacy, cognition, depression/mood, and nutrition. ${ }^{4}$ Recommended interventions included counseling, recommendations for non-pharmacological interventions, pharmacological interventions, and referrals to other specialties or allied healthcare.

One hundred sixty patients with a median age of 80.4 years (standard deviation $=4.2$ ) were randomized to either geriatric consultation plus standard care $(n=60)$ or standard care alone $(n=100)$. Of those randomized to geriatric consultation, 48 (80\%) completed at least one visit with a geriatrician. Consultation did not improve survival at 1 year compared to standard care (difference: $2.9 \%$, $95 \%$ confidence interval $[95 \% \mathrm{CI}]:-9.5 \%$ to $15.2 \%$, $P=0.65)$, and did not significantly reduce the incidence of emergency department visits, hospital admissions, or days in hospital. Consultation did improve the odds of having end-of-life goals-of-care discussions (odds ratio = $3.12,95 \%$ CI: 1.03 to 9.41 ) and was valued by surveyed hematologic oncology clinicians, with $62.9 \%-88.2 \%$ rating consultation as useful in the management of several geriatric domains.

Patient-reported outcomes and quality of life, as well as preserved function (mobility, cognition) and autonomy (ADL, IADL), appear important and likely are not sufficiently surrogated by established study endpoints such as response rates, toxicity and survival outcomes. Assessment of patient-reported outcomes and quality of life studies are both linked to geriatric assessment and are therefore warranted in older patients with hematologic cancer. Patient-related outcomes can help to narrow the gap between patients' and healthcare professionals' view of patients' health and treatment success. ${ }^{7}$ Moreover, several novel drugs have been developed as oral agents, introducing an additional challenge in the management of patients, such as ensuring optimal adherence to therapy in order to maximize treatment efficacy.

In addition to the work presented by DuMontier et al., a recently published review provides updates on the new therapies for common hematologic malignancies with an emphasis on older adult-specific evidence and the evolv- 
ing role of a geriatric assessment in informing therapy selection and management.

\section{Disclosures}

No conflicts of interest to disclose. With regard to work outside this publication, I provide consultancy services for AbbVie, Janssen, AstraZeneca, Beigene, Roche, Kite/Gilead, Celgene/BMS, Takeda, Kyowa-Kirin and ADCTherapeutics. My group has received a research grant from Pfizer.

\section{References}

1. DuMontier C, Uno H, Hshieh T, et al. Randomized controlled trial of geriatric consultation versus standard care in older adults with hematologic malignancies. Haematologica. 2022;107(5):1172-1180.

2. White MC, Holman DM, Boehm JE, et al. Age and cancer risk: a poten- tially modifiable relationship. Am J Prev Med. 2014;46(3 Suppl 1):S7-S15 3. Krok-Schoen JL, Fisher JL, Stephens JA, et al. Incidence and survival of hematological cancers among adults ages $\geq 75$ years. Cancer Med. 2018;7(7):3425-3433

4. Mohile SG, Dale W, Somerfield MR, et al. Practical assessment and management of vulnerabilities in older patients receiving chemotherapy: ASCO guideline for geriatric oncology. J Clin Oncol. 2018;36(22):2326-2347.

5. Abel GA, Klepin HD. Frailty and the management of hematologic malignancies. Blood. 2018;131(5):515-524.

6. Scheepers ERM, Vondeling AM, Thielen N, van der Griend $R$ Stauder R, Hamaker ME. Geriatric assessment in older patients with a hematologic malignancy: a systematic review. Haematologica. 2020;105(6):1484-1493.

7. Cannella L, Efficace F, Giesinger J. How should we assess patientreported outcomes in the onco-hematology clinic? Curr Opin Support Palliat Care. 2018;12(4):522-529.

8. Rosko AE, Cordoba R, Abel G, et al. Advances in management for older adults with hematologic malignancies. J Clin Oncol. 2021;39 (19):2102-2114 